
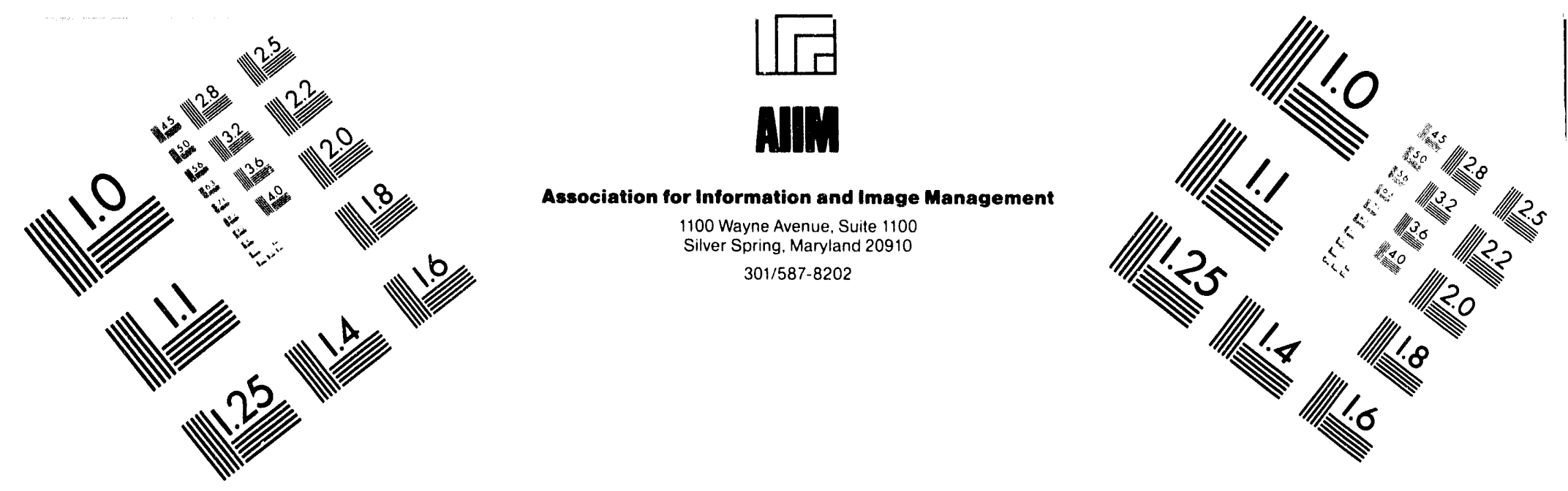

\title{
Centimeter
}

Association for Information and Image Management

1100 Wayne Avenue. Suite 1100

Silver Spring, Maryland 20910

301/587-8202

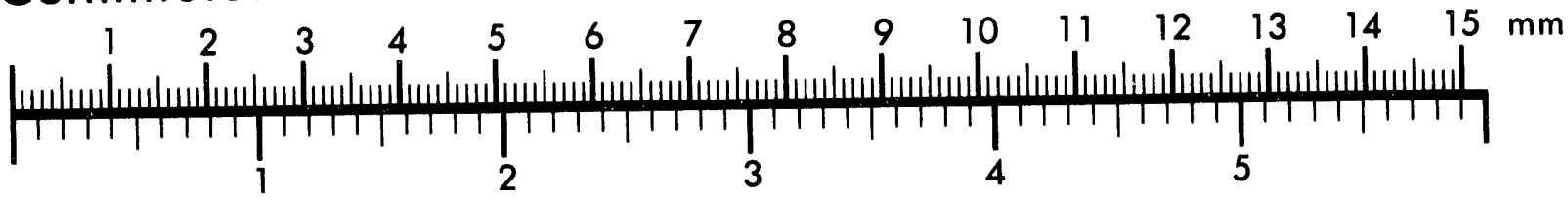
Inches
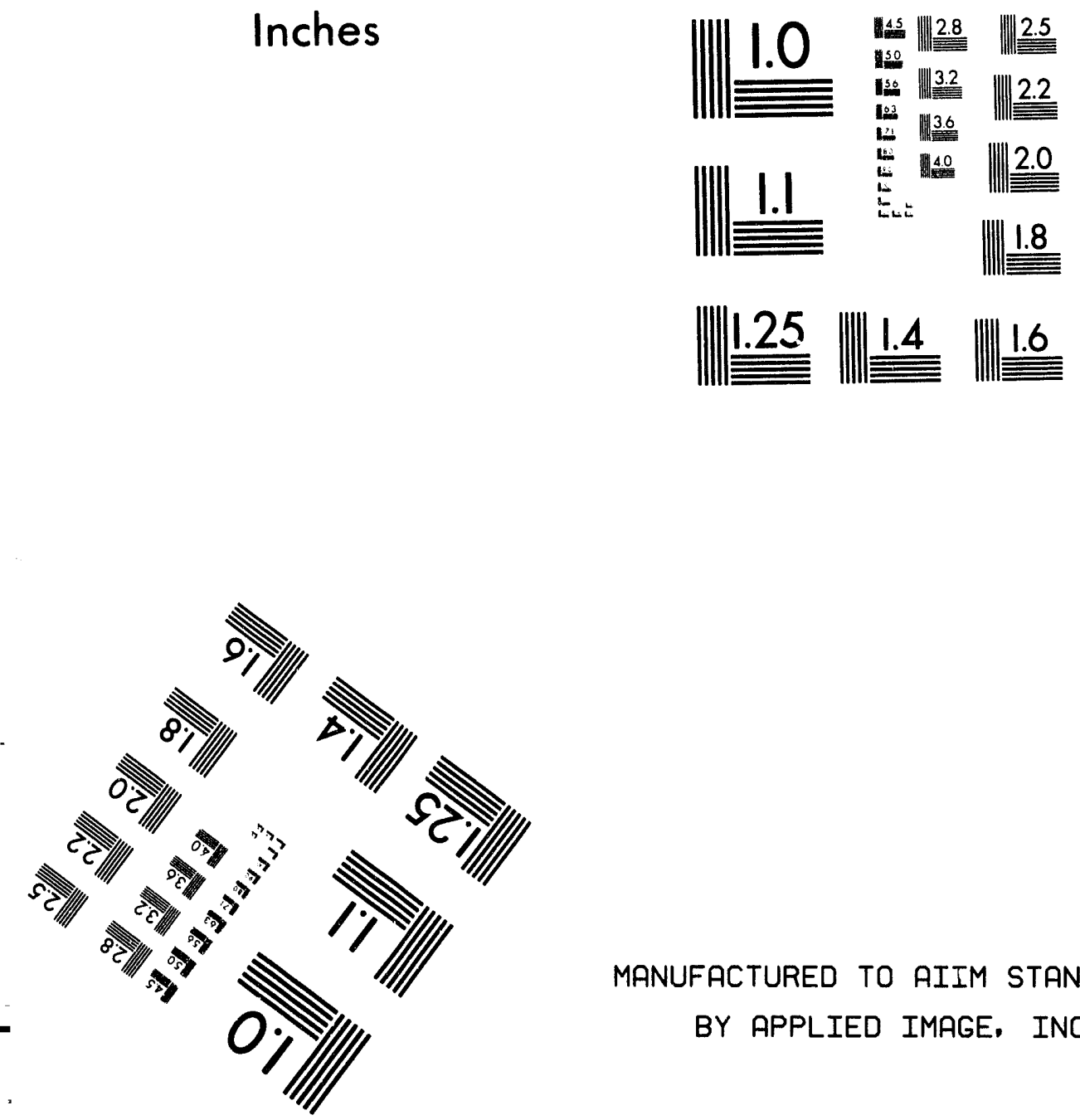

MANUFACTURED TO AIIM STANDARDS

BY APPLIED IMAGE, INC.

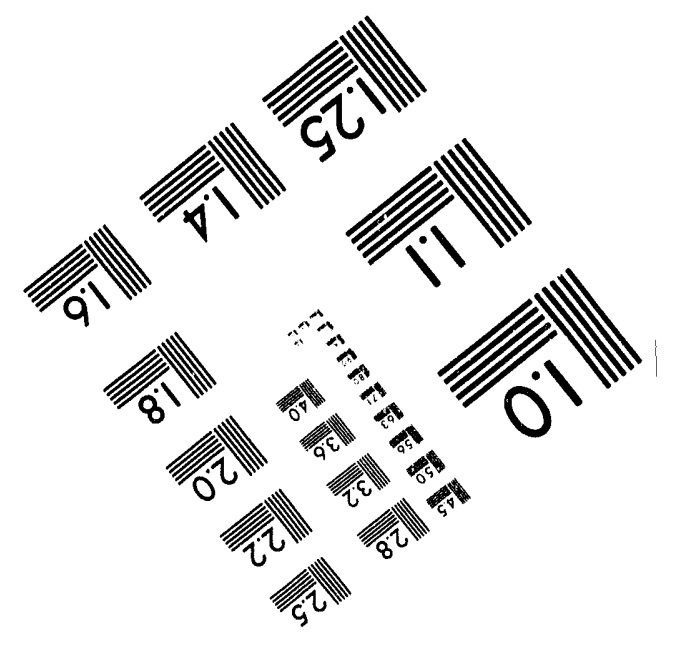



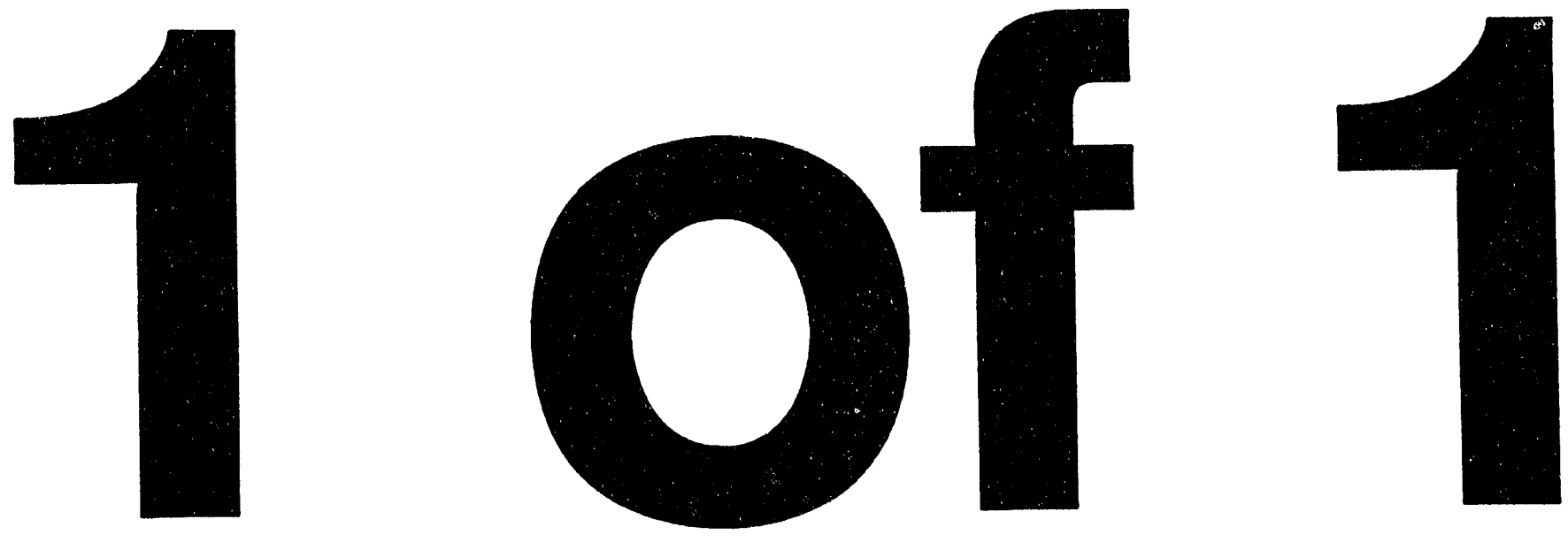


$$
\text { CONF- } 940286--5
$$

\title{
SELECTED PROPERTIES
}

\section{OF IRON ALUMINIDES}

\author{
J. H. Schneibel \\ Oak Ridge National Laboratory \\ Metals and Ceramics Division \\ P. O. Box 2008 \\ Oak Ridge, TN 37831-6114
}

\begin{abstract}
Important properties of iron aluminides have been compiled in order to help engineers and scientists to be able to quickly assess this materials system. This compilation is by no means exhaustive, but it represents a reasonable first effort to summarize the properties of iron aluminides. Considerable care has been used in assembling the data into tables. However, no guarantee can be made that all the values compiled here are correct; and in case of doubt, or in order to obtain more detailed information, the original sources should always be consulted.
\end{abstract}

\section{DISCLAIMER}

This report was prepared as an account of work sponsored by an agency of the United States Government. Neither the United States Governnient nor any agency thereof, nor any of their employees, makes any warranty, express or implied, or assumes any legal liability or responsibility for the accuracy, completeness, or usefulness of any information, apparatus, product, or process disclosed, or represents that its use would not infringe privately owned rights. Reference herein to any specific commercial product, process, or service by trade name, trademark, manufacturer, or otherwise does not necessarily constitute or imply its endorsement, recommendation, or favoring by the United States Government or any agency thereof. The views and opinions of authors expressed herein do not necessarily state or reflect those of the United States Government or any agency thereof. 


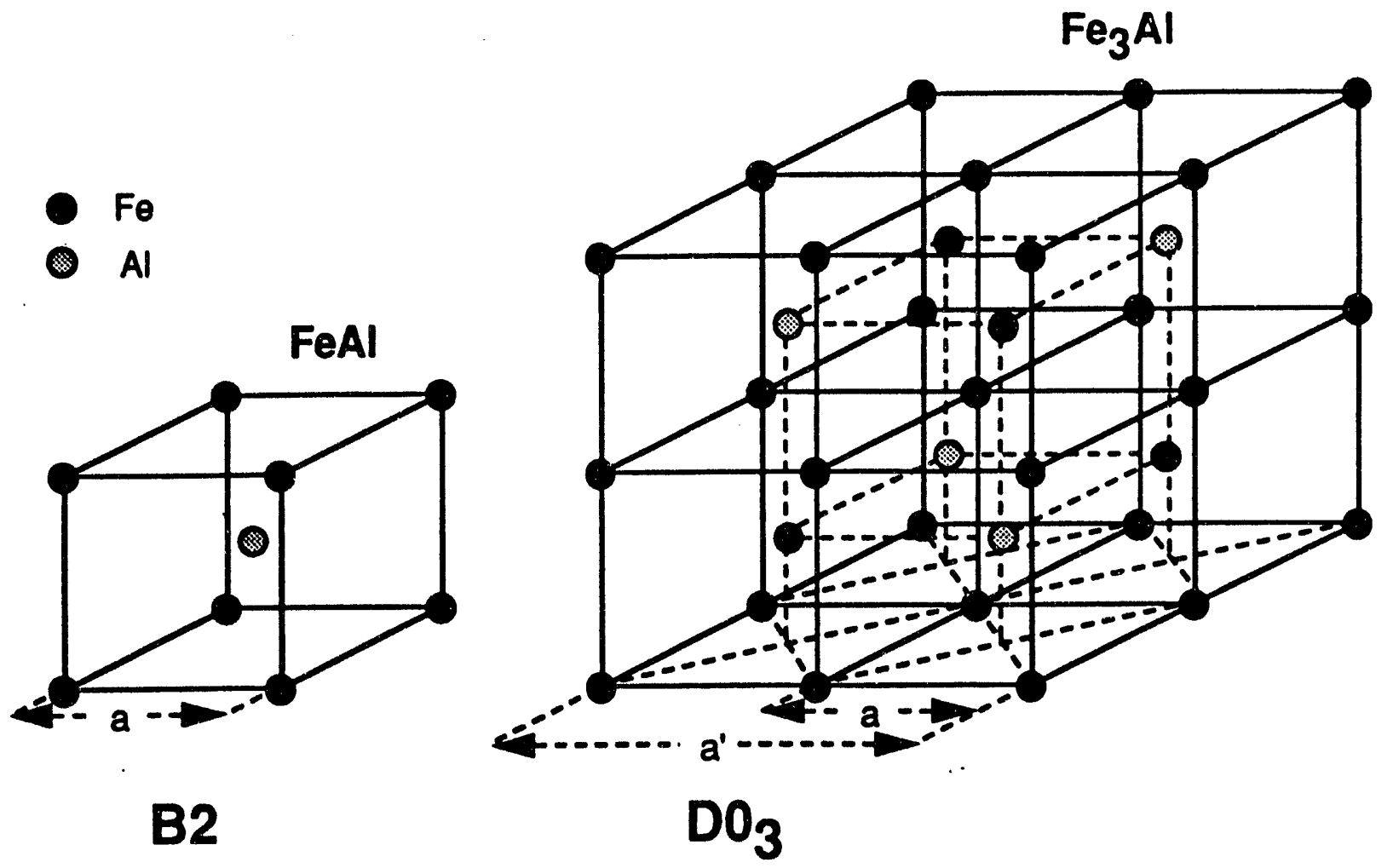

Figure 1 - The $\mathrm{B} 2$ and $\mathrm{DO}_{3}$ crystal structures.

Tuble I Lattice Parameter, a, at Room Temperature, in nm (see also Fig. 1)

\begin{tabular}{|c|c|c|c|c|c|c|c|c|}
\hline \multirow[b]{2}{*}{ Anneal } & \multicolumn{7}{|c|}{$\mathrm{Al}($ at. \%) } & \multirow[b]{2}{*}{ Ref. } \\
\hline & 24.7 & 28.0 & 31.7 & 38.9 & 40 & 45 & 50 & \\
\hline \multirow{3}{*}{$\begin{array}{l}\text { Unspecified } \\
\text { Quenched } \\
\text { from } 1273 \mathrm{~K} \\
\text { Quenched } \\
\text { from } 773 \mathrm{~K}\end{array}$} & 0.28965 & 0.28965 & 0.28946 & 0.28960 & & & & 1 \\
\hline & & & & & 0.2895 & 0.2900 & 0.2905 & 2 \\
\hline & & & & & 0.2896 & 0.2903 & 0.2909 & 2 \\
\hline
\end{tabular}

1. F. Lihl and H. Ebel, quoted in W. B. Pearson, A Handbook of Lattice Spacings and Structures of Metals and Alloys, Vol 2 (Pergamon, Oxford, 1967) p. 560.

2. Y. A. Chang, L. M. Pike, C. T. Liu, A. R. Bilbrey, and D. S. Stone, Intermetallics, 1 (1993), 107.

Table II Densities Derived from Compositions and Lattice Parameters

\begin{tabular}{|l|llll|}
\hline Composition (at. \%) & $\mathrm{Fe}-25 \mathrm{Al}$ & $\mathrm{Fe}-40 \mathrm{Al}$ & $\mathrm{Fe}-45 \mathrm{Al}$ & $\mathrm{Fe}-50 \mathrm{Al}$ \\
\hline Density $\left(\mathrm{Mg} / \mathrm{m}^{3}\right)$ & 6.65 & 6.06 & 5.82 & 5.59 \\
\hline
\end{tabular}


Table III Approximate Melting Temperatures of Iron Aluminides*

\begin{tabular}{|l|lll|}
\hline & \multicolumn{3}{|c|}{ Al (at. \%) } \\
\cline { 2 - 4 } & 25 & 40 & 50 \\
\hline Liquidus (K) & 1789 & 1690 & 1595 \\
Solidus (K) & 1773 & 1649 & 1501 \\
\hline
\end{tabular}

* From Binary Alloy Phase Diagrams. T. B. Massalski, ed.

(American Society for Metals, Metals Park, OH, 1986).

Table IV Electrical Resistivity $\left(10^{-8} \Omega \mathrm{m}\right) *$

\begin{tabular}{|c|ll|}
\hline Temperature $\left({ }^{\circ} \mathrm{C}\right)$ & $\begin{array}{l}\text { Fe-28A1-5Cr-0.05B } \\
\text { (at. \%) }\end{array}$ & $\begin{array}{l}\text { Fe-28Al-5Cr-0.5Nb-0.5Mo- } \\
0.1 \mathrm{Zr}-0.2 \mathrm{~B} \text { (at. \%) }\end{array}$ \\
\hline 20 & 148.5 & 188 \\
200 & 155 & 197 \\
300 & 160 & 201 \\
400 & 164 & 201 \\
500 & 169 & 197 \\
600 & 164 & 193 \\
700 & 162 & 188 \\
800 & 162 & 186 \\
900 & 164 & 186 \\
1000 & 162 & 185 \\
\hline
\end{tabular}

* C. G. McKamey, unpublished results, Oak Ridge National Laboratory.

Table V Thermal Conductivity for Fe-28.1Al-5Cr-0.5Nb-0.2C (at. \%)*

\begin{tabular}{|cl|}
\hline Temperature $\left({ }^{\circ} \mathrm{C}\right)$ & $\begin{array}{l}\text { Thermal Conductivity } \\
{[\mathrm{W} /(\mathrm{m} \mathrm{K})]}\end{array}$ \\
\hline 200 & 13.5 \\
300 & 14.6 \\
400 & 15.8 \\
500 & 17.4 \\
600 & 17.8 \\
700 & 18.4 \\
800 & 19.4 \\
900 & 19.9 \\
1000 & 21.8 \\
1100 & 23.8 \\
\hline
\end{tabular}

* W. D. Porter and P. J. Maziasz, unpublished results, Oak Ridge National Laboratory, August 1993. 
Table VI Thermal Expansion*

\begin{tabular}{|c|c|c|c|c|c|c|}
\hline & $\begin{array}{l}\text { Fe-28Al-5C } \\
\text { (FA-129) }\end{array}$ & Cr-0.5Nb-0.2 & $\mathrm{C}\left(\right.$ at. \%) ${ }^{\dagger}$ & $\begin{array}{l}\mathrm{Fe}-35.8 \mathrm{Al}- \\
\text { (at. \% })^{\dagger \dagger}\end{array}$ & $\begin{array}{l}.2 \mathrm{Mo}-0.05 \mathrm{Z} \\
\mathrm{A}-385)\end{array}$ & $-0.13 \mathrm{C}$ \\
\hline $\mathrm{T}(\mathrm{K})$ & $\begin{array}{l}\text { Thermal } \\
\text { Expansion } \\
\text { (\%), with } \\
\text { respect to } \\
\text { R. T. }\end{array}$ & $\begin{array}{l}\text { Mean } \\
\text { Coefficient } \\
\text { of Thermal } \\
\text { Expansion } \\
(\mathrm{ppm} / \mathrm{K})\end{array}$ & $\begin{array}{l}\text { Instantaneous } \\
\text { Coefficient of } \\
\text { Thermal } \\
\text { Expansion } \\
\text { (ppm/K) }\end{array}$ & $\begin{array}{l}\text { Thermal } \\
\text { Expansion } \\
\text { (\%), with } \\
\text { respect to } \\
\text { R. T. }\end{array}$ & $\begin{array}{l}\text { Mean } \\
\text { Coefficient } \\
\text { of Thermal } \\
\text { Expansion } \\
(\mathrm{ppm} / \mathrm{K})\end{array}$ & $\begin{array}{l}\text { Instantaneous } \\
\text { Coefficient of } \\
\text { Thermal } \\
\text { Expansion } \\
\text { (ppm/K) }\end{array}$ \\
\hline 373 & 0.1239 & 15.4 & 15.9 & 0.1499 & 18.3 & 18.7 \\
\hline 473 & 0.2872 & 15.9 & 17.1 & 0.3414 & 19.0 & 20.2 \\
\hline 573 & 0.4682 & 16.7 & 19.5 & 0.5520 & 19.6 & 21.4 \\
\hline 673 & 0.6804 & 17.9 & 23.1 & 0.7719 & 20.3 & 22.3 \\
\hline 773 & 0.9204 & 19.2 & 25.4 & 0.9896 & 20.6 & 23.2 \\
\hline 873 & 1.1822 & 20.4 & 24.6 & 1.2222 & 21.1 & 24.1 \\
\hline 973 & 1.4277 & 21.0 & 27.3 & 1.4703 & 21.6 & 26.3 \\
\hline 1073 & 1.7037 & 21.8 & 28.7 & 1.7312 & 22.2 & 26.7 \\
\hline 1173 & 2.0058 & 22.8 & 32.7 & 2.0190 & 22.9 & 30.5 \\
\hline 1273 & 2.2924 & 23.4 & 24.5 & 2.3326 & 23.8 & 33.6 \\
\hline 1373 & 2.5235 & 23.4 & 24.1 & 2.6856 & 24.8 & 37.9 \\
\hline 1473 & 2.7700 & 23.5 & 24.7 & 3.1257 & 26.5 & 48.2 \\
\hline
\end{tabular}

* W. D. Porter and P. J. Maziasz, Scr. Metall. Mater, 29 (1993), 1043.

(Note that the vertical axis of Fig. 1 in this reference is scaled incorrectly)

t Note that the $\mathrm{DO}_{3}$ to $\mathrm{B} 2$ and the $\mathrm{B} 2$ to $\alpha$ (disordered Fe-Al solid solution) transitions occur at 816 and $1197 \mathrm{~K}$, respectively

it Note that the B2 to $\alpha$ (disordered Fe-Al solid solution) transition occurs at $1463 \mathrm{~K}$ 
Table VII Solid Solubility Limits of Some Ternary Elements in Iron Aluminides

\begin{tabular}{|lllll|}
\hline Fe & Al (at. \%) & $\begin{array}{l}\text { Ternary Element } \\
(\text { at.\%) }\end{array}$ & $\begin{array}{l}\text { Temperature } \\
(\mathrm{K})\end{array}$ & Ref. \\
\hline $\mathrm{Bal}$ & 28 & $6 \mathrm{Mo}$ & 1123 & 1 \\
$\mathrm{Bal}$ & 40 & $6 \mathrm{Cr}$ & as arc-melted & 2. \\
$\mathrm{Bal}$ & 46 & $25 \mathrm{Cr}$ & 1525 & 3 \\
$\mathrm{Bal}$ & 46 & $25 \mathrm{Co}$ & 1525 & 3 \\
$\mathrm{Bal}$ & 25 & $16 \mathrm{Ti}$ & 1073 & 4 \\
$\mathrm{Bal}$ & 40 & $7.5 \mathrm{Ti}$ & 1073 & 4 \\
\hline
\end{tabular}

1. C. G. McKamey and J. A. Horton, Metall. Trans. A, 20A (1989), 751.

2. P. R. Munroe and I. Baker, Scr. Metall. Mater, 24 (1990), 2273.

3. R. H. Titran, K. M. Vedula, and G. G. Anderson, High-Temperature Ordered Intermetallic Alloys, vol. 39, eds. C. C. Koch, C. T. Liu, and N. S. Stoloff (Pittsburg, PA: Materials Research Society, 1985), p. 309.

4. V. Raghavan, Phase Diagrams of Temary Iron Alloys. Part I, Monograph on Alloy Phase Diagrams (ASM International, Metals Park, OH, 1987).

Table VIII Ceramics which are likely to be Thermodynamically Stable in FeAl at $1273 \mathrm{~K}^{*}$

\begin{tabular}{|llll|}
\hline Carbides & Borides & Oxides & Nitrides \\
\hline $\mathrm{HfC}$ & $\mathrm{HfB}_{2}$ & $\mathrm{Al}_{2} \mathrm{O}_{3}$ & $\mathrm{HfN}$ \\
$\mathrm{TiC}$ & $\mathrm{ScB}_{2}$ & $\mathrm{BeO}$ & \\
$\mathrm{ZCC}$ & $\mathrm{TiB}_{2}$ & $\mathrm{La}_{2} \mathrm{O}_{3}$ & \\
& $\mathrm{ZrB}_{2}$ & $\mathrm{Sc}_{2} \mathrm{O}_{3}$ & \\
& & $\mathrm{Y}_{2} \mathrm{O}_{3}$ & \\
\hline
\end{tabular}

* A. K. Misra, Metall. Trans, A, 21 A (1990), 441.

Table IX Slip Systems in Fe-Al

\begin{tabular}{|lllr|}
\hline Alloy (at. \%) & Temperature (K) & Slip System & Ref. \\
\hline Fe-25Al & 295 & $<111>\{110\}$ & 1 \\
Fe-47Al & 295 & $<111>\{110\}$ & 2 \\
& $>700 \mathrm{~K}$ & $<001>\{100\}$ & 2 \\
\hline
\end{tabular}

1. M. G. Mendiratta, S. K. Ehlers, D. K. Chatterjee, and H. A. Lipsitt, Metall. Trans, 18A (1987), 283.

2. Y. Umakoshi and M. Yamaguchi, Philos. Mag. A, 41 (1980), 573. 
Table X Antiphase Boundary Energies, E, Determined from Four-Fold and Two-Fold Dissociations of Screw Dislocations

\begin{tabular}{|c|c|c|c|c|c|c|}
\hline Alloy (at. \%) & $\begin{array}{l}\text { Crystal } \\
\text { Structure }\end{array}$ & $\begin{array}{l}\mathbf{R}_{\mathrm{NN}^{\dagger}}{ }^{\dagger} \\
(\mathrm{nm})\end{array}$ & $\begin{array}{l}\mathrm{R}_{\mathrm{NNN}}{ }^{\dagger \dagger} \\
(\mathrm{nm})\end{array}$ & $\begin{array}{l}\mathrm{E}_{\mathrm{NN}} \\
\left(\mathrm{mJ} / \mathrm{m}^{2}\right)\end{array}$ & $\begin{array}{l}\mathrm{E}_{\mathrm{NNN}} \\
\left(\mathrm{mJ} / \mathrm{m}^{2}\right)\end{array}$ & Ref. \\
\hline Fe-26.9Al & $\mathrm{DO}_{3}$ & 10 & 23 & 69 & 71 & 1 \\
\hline $\mathrm{Fe}-26.9 \mathrm{Al}$ & B2 & 14 & & 28 & & 1 \\
\hline $\mathrm{Fe}-28 \mathrm{Al}$ & $\mathrm{DO}_{3}$ & 7 & 37 & 76 & 46 & 2 \\
\hline $\mathrm{Fe}-28.3 \mathrm{Al}$ & $\mathrm{DO}_{3}$ & 7 & 25 & 78 & 63 & 1 \\
\hline $\mathrm{Fe}-28.3 \mathrm{Al}$ & B2 & 9 & & 44 & & 1 \\
\hline $\mathrm{Fe}-31.1 \mathrm{Al}$ & B2 & 7 & & 65 & & 1 \\
\hline Fe-33.8Al & B2 & 5 & & 95 & & 1 \\
\hline Fe-39Al & B2 & $4-7+t t$ & & & & 3 \\
\hline $\mathrm{Fe}-28 \mathrm{Al}-4 \mathrm{Cr}$ & $\mathrm{DO}_{3}$ & $15-18$ & 60 & $29-35$ & 28 & 2 \\
\hline $\mathrm{Fe}-28 \mathrm{Al}-4 \mathrm{Cr}$ & $\mathrm{DO}_{3}$ & $6-16$ & $28-34$ & $80-90$ & 60 & 4 \\
\hline
\end{tabular}

$+\mathbf{R}_{\mathbf{N N}}$ is the spacing of the leading or trailing dislocation pairs in the $\mathrm{DO}_{3}$-structure, or the spacing between pairs of partial dislocations in the B2 structure, respectively

it $\mathbf{R}_{\text {NNN }}$ is the spacing between the centers of the leading and trailing pair of a four-fold dislocation in the $\mathrm{DO}_{3}$ structure

t†' Dislocation orientations unspecified

1. R. C. Crawford and I. L. F. Ray, Philos, Mag, 35 (1977), 549.

2. C. G. McKamey, J. A. Horton, and C. T. Liu, J. Mater. Res, 4 (1989), 1156.

3. M. A. Crimp and K. Vedula, Philos. Mag. A, 63 (1991), 559.

4. D. G. Morris, M. M Dadra, and M. A. Morris, Acta Metall. Mater, 41 (1993) 97.

Table XI Elastic Properties at Room Temperature (GPa)

\begin{tabular}{|lllllll|}
\hline Composition (at. \%) & Composition (wt.\%) & $\mathrm{C}_{11}$ & $\mathrm{C}_{12}$ & $\mathrm{C}_{44}$ & $\mathrm{E}$ & Ref. \\
\hline Fe-25 Al & $\mathrm{Fe}-13.87 \mathrm{Al}$ & 171 & 131 & 132 & & 1 \\
$\mathrm{Fe}-28 \mathrm{Al}$ & $\mathrm{Fe}-15.82 \mathrm{Al}$ & 166 & 123 & 131 & & 1 \\
$\mathrm{Fe}-34 \mathrm{Al}$ & $\mathrm{Fe}-19.93 \mathrm{Al}$ & 172 & 114 & 130 & & 1 \\
$\mathrm{Fe}-40 \mathrm{Al}$ & $\mathrm{Fe}-24.36 \mathrm{Al}$ & 181 & 114 & 127 & & 1 \\
$\mathrm{Fe}-31 \mathrm{Al}$ & $\mathrm{Fe}-17.83 \mathrm{Al}$ & & & & 142 & 2 \\
$\mathrm{Fe}-35 \mathrm{Al}$ & $\mathrm{Fe}-20.64 \mathrm{Al}$ & & & & 165 & 2 \\
$\mathrm{Fe}-36 \mathrm{Al}$ & $\mathrm{Fe}-21.37 \mathrm{Al}$ & & & & 180 & 2 \\
$\mathrm{Fe}-38 \mathrm{Al}$ & $\mathrm{Fe}-22.85 \mathrm{Al}$ & & & & 172 & 2 \\
$\mathrm{Fe}-39 \mathrm{Al}$ & $\mathrm{Fe}-23.60 \mathrm{Al}$ & & & & 167 & 2 \\
$\mathrm{Fe}-47 \mathrm{Al}$ & $\mathrm{Fe}-29.99 \mathrm{Al}$ & & & & 164 & 2 \\
$\mathrm{Fe}-50 \mathrm{Al}$ & $\mathrm{Fe}-32.58 \mathrm{Al}$ & - & & & 163 & 2 \\
\hline
\end{tabular}

1. H. J. Leamy, E. D. Gibson, and F. X. Kayser, Acta Metall., 15 (1967), 1827.

2. T. Gödecke and W. Köster, Z. Metallkde., 77 (1986), 408. 
Table XII Yield Strength, Ultimate Tensile Strength, and Ductility of Iron Aluminides at Room Temperature in Laboratory Air ${ }^{\dagger}$

\begin{tabular}{|c|c|c|c|c|c|}
\hline Composition (at. \%) & Processing & $\begin{array}{l}\text { Yield } \\
\text { Stress } \\
(\mathrm{MPa})\end{array}$ & $\begin{array}{l}\text { Ultimate } \\
\text { Tensile } \\
\text { Stress } \\
\text { (MPa) }\end{array}$ & $\begin{array}{l}\text { Fracture } \\
\text { Strain } \\
(\%)\end{array}$ & Ref. \\
\hline $\mathrm{Fe}-27.5 \mathrm{Al}-1.8 \mathrm{Cr}$ & $\begin{array}{l}\text { Elemental powders, reaction } \\
\text { Sintering, } \\
\text { HIP } 1373 \mathrm{~K} / 207 \mathrm{MPa}\end{array}$ & 884 & 1101 & 5 & $\overline{1}$ \\
\hline $\mathrm{Fe}-27.9 \mathrm{Al}-4.6 \mathrm{Cr}$ & $\begin{array}{l}\text { Elemental powders, reaction } \\
\text { sintering, HIP, final anneal } 2 \\
\text { h/1023 K/oil quench }\end{array}$ & 857 & 1095 & 7 & 1 \\
\hline $\mathrm{Fe}-27.9 \mathrm{Al}-4.6 \mathrm{Cr}$ & $\begin{array}{l}\text { Extrusion of prealloyed } \\
\text { powder, final anneal } \\
2\end{array}$ & 358 & 726 & 11.8 & 1 \\
\hline $\mathrm{Fe}-40 \mathrm{Al}-0.02 \mathrm{Z}$ (at. \%) & $\begin{array}{l}\text { Vacuum induction melting } \\
\text { into chilled copper mold, } \\
\text { as-cast }\end{array}$ & & & 1.1 & 2 \\
\hline Fe-28Al-5Cr-0.1Zr-0.04B & $\begin{array}{l}\text { Hot forging and hot rolling } \\
\text { of arc-melted ingot, final } \\
\text { anneal } 1 \mathrm{~h} / 973 \mathrm{~K} / \text { oil quench. } \\
\text { Elongated grains }\end{array}$ & 524 & 1038 & 19 & 3 \\
\hline Fe-28Al & $\begin{array}{l}\text { Casting and hot-rolling, final } \\
\text { anneal } 7 \mathrm{~d} / 773 \mathrm{~K}\end{array}$ & 279 & 514 & 3.7 & 4 \\
\hline $\begin{array}{l}\text { Fe-28Al } \\
\text { Fe-28Al-2Cr }\end{array}$ & $\begin{array}{l}\text { thermomechanical treatment } \\
\text { Casting and hot-rolling, final } \\
\text { anneal } 7 \mathrm{~d} / 773 \mathrm{~K}\end{array}$ & $\begin{array}{l}350 \\
247\end{array}$ & $\begin{array}{l}890 \\
639\end{array}$ & $\begin{array}{r}12.3 \\
9.4\end{array}$ & $\begin{array}{l}5 \\
4\end{array}$ \\
\hline $\begin{array}{l}\mathrm{Fe}-28 \mathrm{Al}-5 \mathrm{Cr} \\
\mathrm{Fe}-28 \mathrm{Al}-6 \mathrm{Cr}\end{array}$ & $\begin{array}{l}\text { thermomechanical treatment } \\
\text { Casting and hot-rolling, final } \\
\text { anneal } 7 \mathrm{~d} / 773 \mathrm{~K}\end{array}$ & $\begin{array}{l}345 \\
232\end{array}$ & $\begin{array}{l}777 \\
535\end{array}$ & $\begin{array}{r}16.8 \\
8.4\end{array}$ & $\begin{array}{l}5 \\
4\end{array}$ \\
\hline $\begin{array}{l}\mathrm{Fe}-28 \mathrm{Al}-5 \mathrm{CrZrMoNbB} \\
\mathrm{Fe}-35.8 \mathrm{Al}\end{array}$ & $\begin{array}{l}\text { thermomechanical treatment } \\
\text { Recrystallization and } 1-2 \mathrm{~h} \text { at } \\
973 \mathrm{~K}\end{array}$ & $\begin{array}{l}530 \\
334\end{array}$ & $\begin{array}{l}950 \\
621\end{array}$ & $\begin{aligned} 14.1 \\
7.6\end{aligned}$ & 6 \\
\hline $\begin{array}{l}\mathrm{Fe}-35.8 \mathrm{Al}-0.2 \mathrm{Mo}-0.05 \mathrm{Zr}- \\
0.24 \mathrm{~B}\end{array}$ & $\begin{array}{l}\text { Recrystallization and } 1-2 \mathrm{~h} \text { at } \\
973 \mathrm{~K}\end{array}$ & 400 & 836 & 11.8 & 6 \\
\hline
\end{tabular}

$\dagger$ Note that strength and ductility are often strongly affected by processing schedule and cooling rate

1. B. H. Rabin and R. N. Wright, Metall. Trans. A, 23A (1992), 35.

2. K.-M. Chang, Met. Trans. A, 21 A (1990), 3027.

3. P. G. Sanders, V. K. Sikka, C. R. Howell, and R. H. Baldwin, Scr. Metall. Mater., 25 (1991), 2365.

4. C. G. McKamey, J. A. Horton, and C. T. Liu, Scripta Metall., 22 (1988), 1679.

5. Z. Q. Sun, Y. D. Huang, W. Y. Yang, and G. L. Chen, in High-Temperature Ordered Intermetallic Alloys V, vol. 288, eds. I. Baker, R. Darolia, J. D. Whittenberger, and M. H. Yoo (Pittsburg, PA: Materials Research Society, 1993), p. 885.

6. P. J. Maziasz, G. M. Goodwin, C. T. Liu, and S. A. David, Scr. Metall. Mater., 27 (1992), 1835. 
Table XIII Critical Resolved Shear Stresses

\begin{tabular}{|c|c|c|c|c|}
\hline $\begin{array}{l}\text { Composition } \\
\text { (at. \%) }\end{array}$ & Anneal & $\begin{array}{l}\text { Test Temperature } \\
(\mathrm{K})\end{array}$ & CRSS (MPa) & Ref. \\
\hline $\begin{array}{l}\mathrm{Fe}-25 \mathrm{Al} \\
\mathrm{Fe}-39 \mathrm{Al}\end{array}$ & $\begin{array}{l}>3.6 \mathrm{ks} / 630 \mathrm{~K} \\
\text { Slow cool from } \\
1273 \mathrm{~K}\end{array}$ & $\begin{array}{l}630 \\
295\end{array}$ & $\begin{array}{l}105-195 \\
97 \pm 13\end{array}$ & $\frac{1}{2}$ \\
\hline $\mathrm{Fe}-39 \mathrm{Al}$ & $\begin{array}{l}\text { Oil quench from } \\
1273 \mathrm{~K}\end{array}$ & 295 & $304 \pm 16$ & 2 \\
\hline $\begin{array}{l}\mathrm{Fe}-47 \mathrm{Al} \\
\mathrm{Fe}-49 \mathrm{Al}\end{array}$ & $\begin{array}{l}24 \mathrm{~h} / 1123 \mathrm{~K} / \text { slow cool } \\
\text { Slow cool from } \\
1273 \mathrm{~K}\end{array}$ & $\begin{array}{l}470 \\
295\end{array}$ & $\begin{array}{l}375 \\
202 \pm 17\end{array}$ & $\begin{array}{l}3 \\
2\end{array}$ \\
\hline $\mathrm{Fe}-49 \mathrm{Al}$ & $\begin{array}{l}\text { Oil quench from } \\
1273 \mathrm{~K}\end{array}$ & 295 & $318 \pm 16$ & 2 \\
\hline
\end{tabular}

1. S. Hanada, S. Watanabe, T. Sato, and O. Izumi, Scr. Metall, 15 (1981), 1345.

2. M. A. Crimp and K. Vedula, Philos. Mag, A, 63 (1991), 559.

3. Y. Umakoshi and M. Yamaguchi, Philos. Mag. A, 41 (1980) 573.

Table XIV Work Hardening Rates of Iron Aluminides at Room Temperature and a Strain of $1 \%$

\begin{tabular}{|llll|}
\hline Alloy (at. \%) & $\begin{array}{l}\text { Yield Strength } \\
(\mathrm{MPa})\end{array}$ & $\begin{array}{l}\text { Work Hardening } \\
\text { Rate (GPa) }\end{array}$ & Ref. \\
\hline Fe-28Al & 360 & 6 & 1 \\
& 600 & 1 & 1 \\
Fe-28Al-4Cr & 225 & 3.7 & 1 \\
Fe-31Al & 560 & 6.9 & 2 \\
\hline
\end{tabular}

1. D. G. Morris, M. M. Dadras, and M. A. Morris, Acta Metall. Mater., 41 (1993), 97.

2. M. G. Mendiratta, S. K. Ehlers, D. K. Chatterjee, and H. A. Lipsitt, Metall. Trans. A, 18A (1987), 283. 
Table XV Effect of different Environments on Tensile Ductility (\%) of Iron Aluminides at Room Temperature

\begin{tabular}{|c|c|c|c|c|c|c|c|c|}
\hline $\begin{array}{l}\text { Composition } \\
\text { (at.\%) }\end{array}$ & $\begin{array}{l}\text { Final } \\
\text { Treatment }\end{array}$ & $\begin{array}{l}\mathrm{H}_{2} \mathrm{O} \\
\text { Vapor }\end{array}$ & Lab Air & $\mathrm{Ar}+4 \% \mathrm{H}_{2}$ & $\begin{array}{l}\text { Silicone } \\
\text { Oil } \\
\text { Coating, } \\
\text { in Air }\end{array}$ & $\begin{array}{l}\text { Vacuum } \\
\left(=1 \times 10^{-4}\right. \\
\mathrm{Pa})\end{array}$ & Oxygen & Ref. \\
\hline $\mathrm{Fe}-24.6 \mathrm{Al}$ & Assorted & & $0.7-6.4$ & & & & $3.2-9.3$ & 1 \\
\hline $\mathrm{Fe}-28 \mathrm{Al}$ & $5 \mathrm{~d} / 773 \mathrm{~K}$ & 2.1 & 3.7 & & & 12.4 & $11.7^{\dagger}$ & 2 \\
\hline $\mathrm{Fe}-28 \mathrm{Al}$ & $2 \mathrm{~h} / 973 \mathrm{~K}$ & 2.1 & 4.1 & $8.4^{\dagger}$ & & 12.8 & $12.0^{\dagger}$ & 2 \\
\hline Fe-33Al & $1 \mathrm{~h} / 873 \mathrm{~K}$ & & 2.9 & & & & 11.2 & 1 \\
\hline $\mathrm{Fe}-36.5 \mathrm{Al}$ & $2 \mathrm{~h} / 973 \mathrm{~K}$ & 2.4 & 2.2 & $6.1^{t+t}$ & 5.6 & 5.4 & $11.3^{\dagger t}$ & 3 \\
\hline $\mathrm{Fe}-36.5 \mathrm{Al}$ & $2 \mathrm{~W} / 973 \mathrm{X}$ & & & & & & $17.6^{*}$ & 4 \\
\hline Fe-40Al & $2 \mathrm{~h} / 973 \mathrm{~K}$ & & 1.2 & & & & $3.2^{\#}$ & 4 \\
\hline $\mathrm{Fe}-40 \mathrm{Al}-0.1 \mathrm{~B}$ & $2 \mathrm{~h} / 973 \mathrm{~K}$ & & 4.3 & & & & $16.8^{*}$ & 4 \\
\hline \multicolumn{9}{|l|}{$\begin{array}{l}+67 \mathrm{KPa} \\
+\dagger 67 \mathrm{~Pa} \\
+\dagger 110 \mathrm{~Pa} \\
+6.7 \times 10^{-4} \mathrm{~Pa}\end{array}$} \\
\hline \multicolumn{9}{|c|}{$\begin{array}{l}\text { 1. R. J. Lynch, K. A. Gee, and L. A. Heldt, Scr. Metall, Mater, } 30 \text { (1994), } 945 \text {. } \\
\text { 2. C. T. Liu, C. G. McKamey, and E. H. Lee, Scr. Metall. Mater, } 24 \text { (1990), } 385 . \\
\text { 3. C. T. Liu, E. H. Lee, and C. G. McKamey, Scr. Metall, } 23 \text { (1989), } 875 \text {. } \\
\text { 4. C. T. Liu and E. P. George, Scr. Metall. Mater., } 24 \text { (1990), } 1285 .\end{array}$} \\
\hline
\end{tabular}

Table XVI Tensile Properties of Fe-28Al-5Cr-0.5Nb-0.2C (FA-129) at Different Temperatures*

\begin{tabular}{|rccc|}
\hline $\mathrm{T}\left({ }^{\circ} \mathrm{C}\right)$ & $\begin{array}{l}\text { Yield Strength } \\
(\mathrm{MPa})\end{array}$ & $\begin{array}{l}\text { Ultimate Tensile } \\
\text { Strength }(\mathrm{MPa})\end{array}$ & $\begin{array}{l}\text { Elongation to } \\
\text { Fracture (\%) }\end{array}$ \\
\hline 20 & 412 & 824 & 14 \\
93 & 378 & 954 & 15 \\
204 & 357 & 1016 & 30 \\
316 & 371 & 892 & 28 \\
427 & 391 & 783 & 26 \\
538 & 371 & 570 & 33 \\
593 & 343 & 467 & 42 \\
649 & 295 & 343 & 58 \\
704 & 220 & 240 & 80 \\
760 & 124 & 137 & 108 \\
\hline
\end{tabular}

* V. K. Sikka, data package on $\mathrm{Fe}_{3} \mathrm{Al}$ - and $\mathrm{Fe}-\mathrm{Al}$-based alloys developed at ORNL, Oak Ridge National Laboratory, January 20, 1993. 
Table XVII Tensile Properties of Fe-35.8Al-0.2Mo-0.05Zr-0.24B (at.\%) at Different Temperatures*

\begin{tabular}{|rlll|}
\hline $\mathrm{T}\left({ }^{\circ} \mathrm{C}\right)$ & $\begin{array}{l}\text { Yield Strength } \\
(\mathrm{MPa})\end{array}$ & $\begin{array}{l}\text { Ulimate Tensile } \\
\text { Stength (MPa) }\end{array}$ & $\begin{array}{l}\text { Elongation to } \\
\text { Fracture (\%) }\end{array}$ \\
\hline 24 & 351,386 & 569,636 & $6.3,6.4$ \\
100 & 358,336 & 478,449 & $2.6,3.0$ \\
200 & 357 & 677 & 8.6 \\
300 & 396 & 643 & 7.4 \\
400 & 346 & 464 & 3.5 \\
500 & 365 & 424 & 2 \\
600 & 297 & 381 & 21.3 \\
700 & 223 & 260 & 24.1 \\
800 & 93 & 132 & 66.3 \\
\hline
\end{tabular}

*C. T. Liu, V. K. Sikka, and C. G. McKamey, ORNL/TM-12199, February 1993.

Table XVIII Charpy Impact Energy (J) for Iron Aluminides as a Function of Temperature

\begin{tabular}{|c|c|c|c|c|c|c|c|}
\hline \multirow{7}{*}{$\begin{array}{l}\text { Alloy (at. \%) } \\
\text { Fe-40Al-0.02Zr } \\
\text { Directionally solidified, as-cast } \\
\text { Fe-28Al-5Cr-0.5Nb-0.2C (FA-129) } \\
\text { Rolled and annealed (750 }(75) \\
\text { Fe-39.5Al-0.05Zr, extruded, } \\
\text { bimodal grain size } \\
\text { Fe-39.5Al-0.05Zr, extruded, } \\
\text { equiaxed grains } \\
\text { Fe-41Al-1.7Cr-0.03Ce, extruded, } \\
\text { bimodal grain size } \\
\text { Fe-41Al-1.7Cr-0.03Ce, extruded, }\end{array}$} & $173 \mathrm{~K}$ & $295 \mathrm{~K}$ & $473 \mathrm{~K}$ & $673 \mathrm{~K}$ & $873 \mathrm{~K}$ & $1073 \mathrm{~K}$ & Ref. \\
\hline & & 76 & 42 & 22 & 10 & 2.7 & 1 \\
\hline & 3 & 6 & 9 & 29 & 37 & 36 & 2 \\
\hline & & $55 \pm 3$ & & & & & 3 \\
\hline & & $39 \pm 4$ & & & & & 3 \\
\hline & & $53 \pm 5$ & & & & & 3 \\
\hline & & $6 \pm 1$ & & & & & 3 \\
\hline
\end{tabular}

1. K.-M. Chang, Met. Trans, A, 21A (1990), 3027.

2. B. G. Gieseke, D. J. Alexander, V. K. Sikka, and R. H. Baldwin, Scr. Metall. Mater, 29 (1993), 129.

3. D. Pocci, O. Tassa, and C. Testani, these proceedings. 
Table XIX Fracture Toughness of Iron Aluminides

\begin{tabular}{|c|c|c|c|c|c|}
\hline $\begin{array}{l}\text { Alloys (at. \%) } \\
\text { (polycrystalline } \\
\text { unless noted } \\
\text { otherwise) }\end{array}$ & $\begin{array}{l}\mathrm{K}_{\mathrm{Ic}} \text { or } \mathrm{K} \\
\left(\mathrm{MPa} \mathrm{m}^{1 / 2}\right)\end{array}$ & $\begin{array}{l}\text { Heat } \\
\text { Treatment }\end{array}$ & Test Method & $\begin{array}{l}\text { Average } \\
\text { Crack } \\
\text { Velocity } \\
(\mu \mathrm{m} / \mathrm{s})\end{array}$ & Ref. \\
\hline Fe-40Al, Single Crystal & $33-56$ & as-solidified & $\begin{array}{l}\text { Single-edge notch, } \\
\text { 4-point bend test }\end{array}$ & & 1 \\
\hline $\begin{array}{l}\text { Fe-48Al, Directionally } \\
\text { solidified }\end{array}$ & 12 & as-solidified & $"$ & & 1 \\
\hline $\mathrm{Fe}-40 \mathrm{Al}$ & $=40$ & $\begin{array}{l}1273 \mathrm{~K} / \text { fast cool } \\
\text { in vacuum }\end{array}$ & $\begin{array}{l}\text { Chevron-notch } \\
\text { 3-point bend test }\end{array}$ & 300 & 2 \\
\hline Fe-40Al-0.1B & $\approx 50$ & $\begin{array}{l}1273 \mathrm{~K} / \text { fast cool } \\
\text { in vacuum }\end{array}$ & $"$ & 200 & 2 \\
\hline Fe-45Al & $=12$ & $\begin{array}{l}1273 \mathrm{~K} / \text { fast cosl } \\
\text { in vacuum }\end{array}$ & $"$ & 700 & 2 \\
\hline $\mathrm{Fe}-4 \mathrm{JAl}$ & $11-16$ & $5 d / 673 K$ & Fatigue pre-crack & "slow" & 3 \\
\hline $\mathrm{Fe}-45 \mathrm{Al}$ & $25-27$ & $5 \mathrm{~d} / 673 \mathrm{~K}$ & Fatigue pre-crack & "fast" & 3 \\
\hline
\end{tabular}

1. K.-M. Chang, R. Darolia, and H. A. Lipsitt, Acta Metall. Mater., 40 (1992), 2727.

2. J. H. Schneibel, M. G. Jenkins, and P. J. Maziasz, High-Temperature Ordered Intermetallic Alloys V, vol. 288, eds. I. Baker, R. Darolia, J. D. Whittenberger, and M. H. Yoo (Pittsburgh, PA: Materials Research Society, 1993), 549.

3. O. Klein, P. Nagpal, and I. Baker, ibid., p. 935.

Table XX Creep Data for FezAl-Based Iron Aluminides at an Applied Stress of $138 \mathrm{MPa}^{*}$

\begin{tabular}{|clllll|}
\hline $\begin{array}{l}\text { Composition } \\
\text { (at. \%) }\end{array}$ & $\begin{array}{l}\text { Temperature } \\
(\mathrm{K})\end{array}$ & Creep Life (h) & $\begin{array}{l}\text { Minimum Creep } \\
\text { Rate }\left(\mathrm{s}^{-1}\right)\end{array}$ & $\begin{array}{l}\text { Elongation to } \\
\text { Fracture (\%) }\end{array}$ & Ref. \\
\hline Fe-28Al & 823 & 87 & $6.1 \times 10^{-7}$ & 30 & 1 \\
$"$ & 873 & 5.8 & $1.3 \times 10^{-5}$ & 34 & 1 \\
$"$ & 923 & 0.6 & $8.5 \times 10^{-5}$ & 37 & 1 \\
Fe-28Al-2Mo & 873 & 835 & $1.1 \times 10^{-7}$ & 40 & 1 \\
$"$ & 923 & 50 & $1.3 \times 10^{-6}$ & 44 & 1 \\
Fe-28Al-1Nb & 873 & $>1975$ & $1.9 \times 10^{-9}$ & $>1$ & 1 \\
$" 1$ & 923 & 304 & $1.4 \times 10^{-8}$ & 37 & 1 \\
Fe-35.8Al & 866 & 46.4 & $6.4 \times 10^{-7}$ & 28 & 2 \\
Fe-35.8Al-0.2Mo- & 866 & 932 & $8.3 \times 10^{-8}$ & 74 & 2 \\
$0.05 \mathrm{Zr}-0.24 \mathrm{~B}$ & & & & & \\
\hline
\end{tabular}

1. C. G. McKamey, P. J. Maziasz, and J. W. Jonts, J. Mater. Res., 7 (1992), 2089.

2. P. J. Maziasz, G. M. Goodwin, C. T. Liu, and S. A. David, Scr. Metall. Mater., 27 (1992), 1835. 
Table XXI $1000 \mathrm{~h}$ Rupture Strengths for Fey Al-Based Iron Aluminides*

\begin{tabular}{|l|lll|}
\hline Composition (at. \%) & $823 \mathrm{~K}$ & $873 \mathrm{~K}$ & $923 \mathrm{~K}$ \\
\hline Fe-28.1Al-2Cr-0.04B & 98 & 55 & 31 \\
Fe-28Al-5Cr-0.08Zr-0.04B & 117 & 65 & 36 \\
Fe-28.1Al-5Cr-0.5Nb-0.2C & 149 & 77 & 40 \\
\hline
\end{tabular}

*V. K. Sikka and R. H. Baldwin, SAMPE Quart, 24, No 1 (1992), 2.

Table XXII Stresses Needed to Produce a Secondary Creep Rate of $10^{-7} \mathrm{~s}^{-1}$ in FeAl-Based Iron Aluminides

\begin{tabular}{|l|lllll|}
\hline Composition (at. \%) & $1023 \mathrm{~K}$ & $1100 \mathrm{~K}$ & $1173 \mathrm{~K}$ & $1200 \mathrm{~K}$ & Ref. \\
\hline $\mathrm{Fe}-48.7 \mathrm{Al}$ & & $12 \mathrm{MPa}$ & & $7 \mathrm{MPa}$ & 1 \\
re-40Al & & & & $5 \mathrm{MPa}$ & 1 \\
$\mathrm{Fe}-40 \mathrm{Al}-2$ vol. \% $\mathrm{Y}_{2} \mathrm{O}_{3}$ & & & & $22 \mathrm{MPa}$ & 2 \\
$\mathrm{Fe}-10 \mathrm{Ni}-50 \mathrm{Al}$ & $100 \mathrm{MPa}$ & & 15 & & 3 \\
\hline
\end{tabular}

1. J. D. Whittenberger, Mater. Sci. Eng., 77 (1986), 103.

2. J. H. Schneibel, P. Grahle, and J. Rösler, Mater. Sci. Eng., A 153 (1992), 6,84.

3. I. Jung, M. Rudy, and G. Sauthoff, in High-Temperature Ordered Intermetallic Alloys II, vol. 81, eds. N. S. Stoloff, C. C. Koch, C. T. Liu, and O. Izumi (Pittsburgh, PA: Materials Research Society, 1987), 263. 
Table XXIII Weight Changes for Iron Aluminides Exposed to Iothermal Oxidizing Environments*

\begin{tabular}{|lcccc|}
\hline $\begin{array}{l}\text { Alloy } \\
\text { (at.\%) }\end{array}$ & Environment & $\begin{array}{c}\text { Temperature } \\
\text { ( C) }\end{array}$ & $\begin{array}{c}\text { Time } \\
\text { (h) }\end{array}$ & $\begin{array}{c}\text { Weight Change } \\
\left(\mathrm{mg} / \mathrm{cm}^{2}\right)\end{array}$ \\
\hline Fe-22Al & O2, 0.15 bar & 725 & 6 & .004 \\
Fe-22Al & O2, 0.15 bar & 775 & 6 & .007 \\
Fe-22Al & O2, 0.15 bar & 825 & 6 & .015 \\
Fe-25Al & Air & 816 & 240 & $-0.35 a$ \\
Fe-28Al & Air & 800 & 25 & 0.05 \\
Fe-28Al & Air & 800 & 114 & 0.06 \\
Fe-28Al & Air & 900 & 100 & 0.08 \\
Fe-28Al & Air & 900 & 100 & 0.15 \\
Fe-28Al-4Cr & Air & 800 & 25 & 0.04 \\
Fe-28Al-4Cr & Air & 800 & 100 & 0.12 \\
Fe-28Al-5Cr-0.1Zr & Air & 800 & 24 & 0.17 \\
Fe-28Al-5Cr-0.1Zr & Air & 900 & 25 & 0.14 \\
Fe-28Al-5Cr-0.1Zr & Air & 900 & 100 & 0.16 \\
Fe-35Al & Air & 800 & 100 & 0.07 \\
\hline
\end{tabular}

* P. F. Tortorelli and J. H. DeVan in Section 3 from Chapter 3, "Oxidation and Other Corrosion Phenomena At Intermediate Temperatures $\left(300-800^{\circ} \mathrm{C}\right)$, " in Comosion and Oxidation of Intermetallic Alloys, G. Welsch and P. D. Desai (editors), DoD Metals Information Analysis Center, to be published in 1994.

a After cooling from exposure temperature

† P. F. Tortorelli, unpublished Research, Oak Ridge National Laboratory

Table XXIV Weight Gains of $\mathrm{Fe}_{3} \mathrm{Al}$ Exposed to $\mathrm{N}_{2}-1 \% \mathrm{O}_{2}-0.5 \% \mathrm{SO}_{2} *$

\begin{tabular}{|ccc|}
\hline $\begin{array}{c}\text { Temperature } \\
\text { (C) }\end{array}$ & Salt Deposit $^{a}$ & $\begin{array}{c}\text { Weight Gain } \\
\text { After } 96 \mathrm{~h}\left(\mathrm{mg} / \mathrm{cm}^{2}\right)\end{array}$ \\
\hline 600 & none & $<0.1$ \\
700 & none & 0.6 \\
800 & none & 0.4 \\
600 & $\mathrm{Na}_{2} \mathrm{SO}_{4}-10 \mathrm{wt} \% \mathrm{NaCl}$ & 4.6 \\
700 & $\mathrm{Na}_{2} \mathrm{SO}_{4}-10 \mathrm{wt} \% \mathrm{NaCl}$ & 1.9 \\
800 & $\mathrm{Na}_{2} \mathrm{SO}_{4}-10 \mathrm{wt} \% \mathrm{NaCl}$ & 2.3 \\
\hline
\end{tabular}

* P. F. Tortorelli and J. H. DeVan in Section 3 from Chapter 3, "Oxidation and Other Corrosion Phenomena At Intermediate Temperatures $\left(300-800^{\circ} \mathrm{C}\right)$, " in Comosion and Oxidation of Intermetallic Alloys, G. Welsch and P. D. Desai (editors), DoD Metals Information Analysis Center, to be published in 1994. 
This research was sponsored by the Division of Materials Sciences, U.S. Department of Energy, under contract DE-ACO5-84OR21400 with Martin Marietta Energy Systems, Inc. The author would like to thank C. G. McKamey and P. J. Maziasz for their review of the manuscript and helpful comments. Thanks go also to W. D. Porter, C. G. McKamey, and P. F. Tortorelli for providing unpublished data for incorporation in this compilation. 

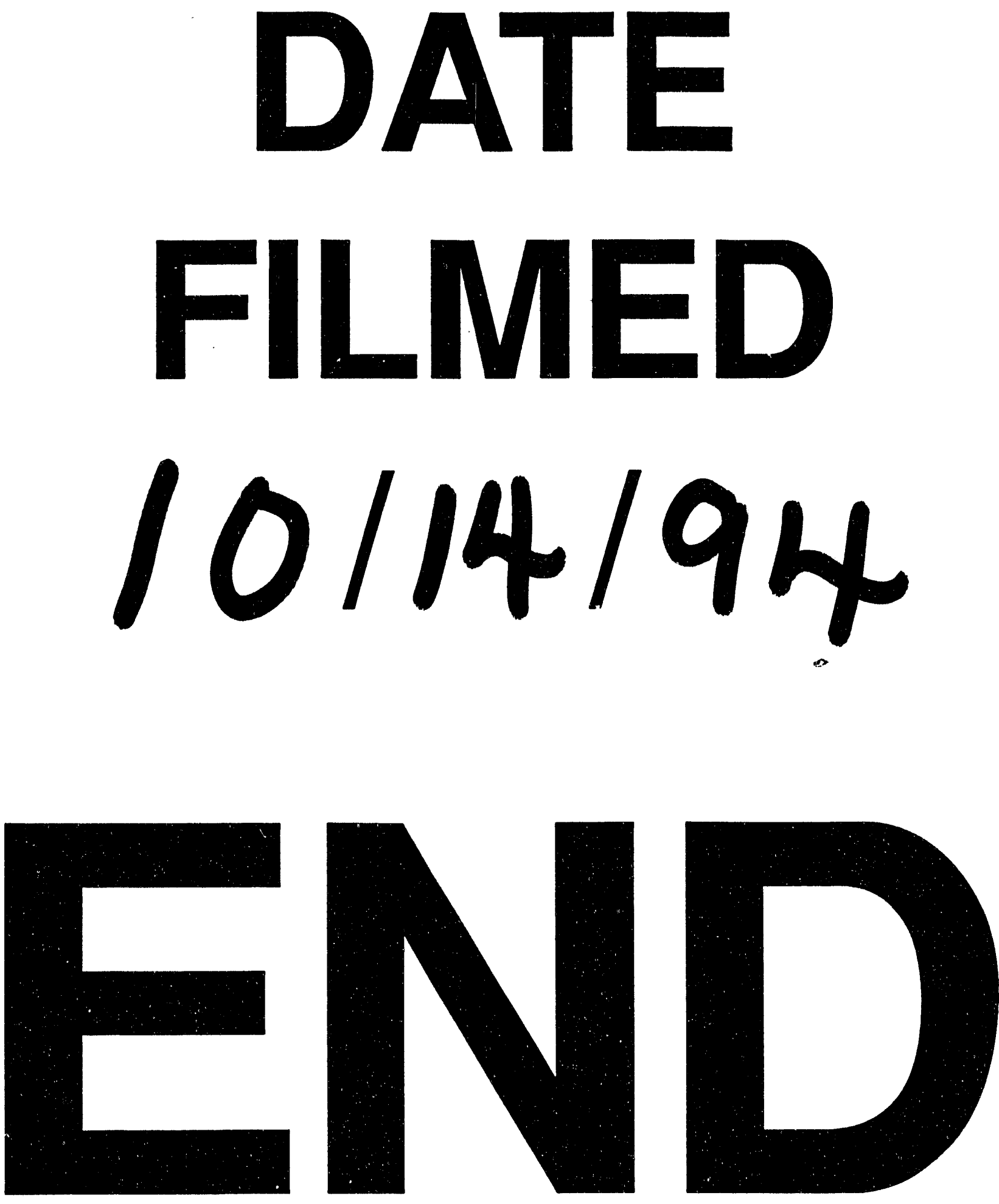
